\title{
Diffuse optical tomography to investigate the newborn brain
}

\author{
Chuen Wai Lee ${ }^{1,2}$, Robert J. Cooper ${ }^{1,3}$ and Topun Austin ${ }^{1,2}$
}

Over the past 15 years, functional near-infrared spectroscopy (fNIRS) has emerged as a powerful technology for studying the developing brain. Diffuse optical tomography (DOT) is an extension of fNIRS that combines hemodynamic information from dense optical sensor arrays over a wide field of view. Using image reconstruction techniques, DOT can provide images of the hemodynamic correlates to neural function that are comparable to those produced by functional magnetic resonance imaging. This review article explains the principles of DOT, and highlights the growing literature on the use of DOT in the study of healthy development of the infant brain, and the study of novel pathophysiology in infants with brain injury. Current challenges, particularly around instrumentation and image reconstruction, will be discussed, as will the future of this growing field, with particular focus on whole-brain, time-resolved DOT.

$\mathbf{T}$ he field of medical physics and biomedical engineering has provided a range of novel neuroimaging techniques that have enabled scientists and clinicians to investigate the functional organization and architecture of the human brain in health and disease. In recent years, functional near-infrared spectroscopy (fNIRS) has become an increasingly valuable tool. In addition to being non-invasive, relatively low-cost and easy to set up, it can be carried out in infants who are awake and do not need to be transported to specialized imaging facilities. Diffuse optical tomography (DOT) is a natural extension of fNIRS, which combines multi-channel data acquisition with image reconstruction software to provide images of changes in regional blood volume and oxygenation at high temporal and spatial resolution. This technology is increasingly being used by researchers and clinicians to study healthy brain development, as well as pathophysiology in critically ill infants in intensive care. Figure 1 summarizes the terminology and NIRS domains that will be described in this paper.

The aim of this paper is to explain the principles of DOT and highlight studies using DOT in newborn infants. We also summarize the current challenges of the technique and discuss the future of the field, with particular emphasis on technological developments and time-domain DOT (TDDOT) techniques.

\section{PRINCIPLES OF OPTICAL IMAGING \\ Near-Infrared Spectroscopy}

NIRS is a non-invasive tissue-monitoring technique that exploits the relative transparency of biological tissue to near-infrared (NIR) light $(650-950 \mathrm{~nm})$ and the wavelength-dependent absorption characteristics of light-absorbing compounds or chromophores. In the brain, hemoglobin is the dominant chromophore, whose absorption varies with oxygenation-first described by Jöbsis (1-3) (Figure 2a).

However, the dominant interaction between NIR light and tissue is not absorption but scattering, such that the path traveled by a given photon will constitute a 'random walk': bouncing randomly from one scattering event to the next. NIR light therefore forms a diffuse field when entering tissue. Assuming that scattering remains constant, loss of light, or attenuation, will be because of changes in absorption by the main chromophores (light-absorbing molecules). The simplest form of NIRS measurement consists of two wavelengths of NIR light, coupled into tissue via an optical fiber positioned a few centimeters away to collect light and transmit it to a detector. This method has been used extensively to study cerebral oxygenation in the newborn infant $(4,5)$ (for a review, see (ref. 6)).

\section{Functional Near-Infrared Spectroscopy}

During cortical activation, neural excitation produces an increased metabolic demand that results in local changes in oxygen consumption, vasodilatation, increased blood flow, and increased oxygenation. This relationship, between neural activity and vasodilation, is known as neurovascular coupling. In adults, a local increase in neural activity is typically characterized by an increase in oxyhemoglobin concentration and a decrease in deoxyhemoglobin concentration, because of the rapid influx of oxygenated blood $(7,8)$. Although the exact mechanisms of neurovascular coupling are still a matter of active investigation, the consistency of the functional hemodynamic response means that it provides an effective indirect measure of cortical function. As NIRS is able to monitor changes in the concentration of oxyhemoglobin and

\footnotetext{
${ }^{1}$ neoLAB, The Evelyn Perinatal Imaging Centre, The Rosie Hospital, Cambridge University Hospitals NHS Foundation Trust, Cambridge, UK; ${ }^{2}$ Department of Neonatology, The Rosie Hospital, Cambridge University Hospitals NHS Foundation Trust, Cambridge, UK; ${ }^{3}$ Department of Medical Physics and Biomedical Engineering, University College London, London, UK. Correspondence: Topun Austin (topun.austin@addenbrookes.nhs.uk)

Received 18 July 2016; accepted 6 February 2017; advance online publication 31 May 2017. doi:10.1038/pr.2017.107
} 


\section{Diffuse optical tomography in newborns $\mid$ ReView}

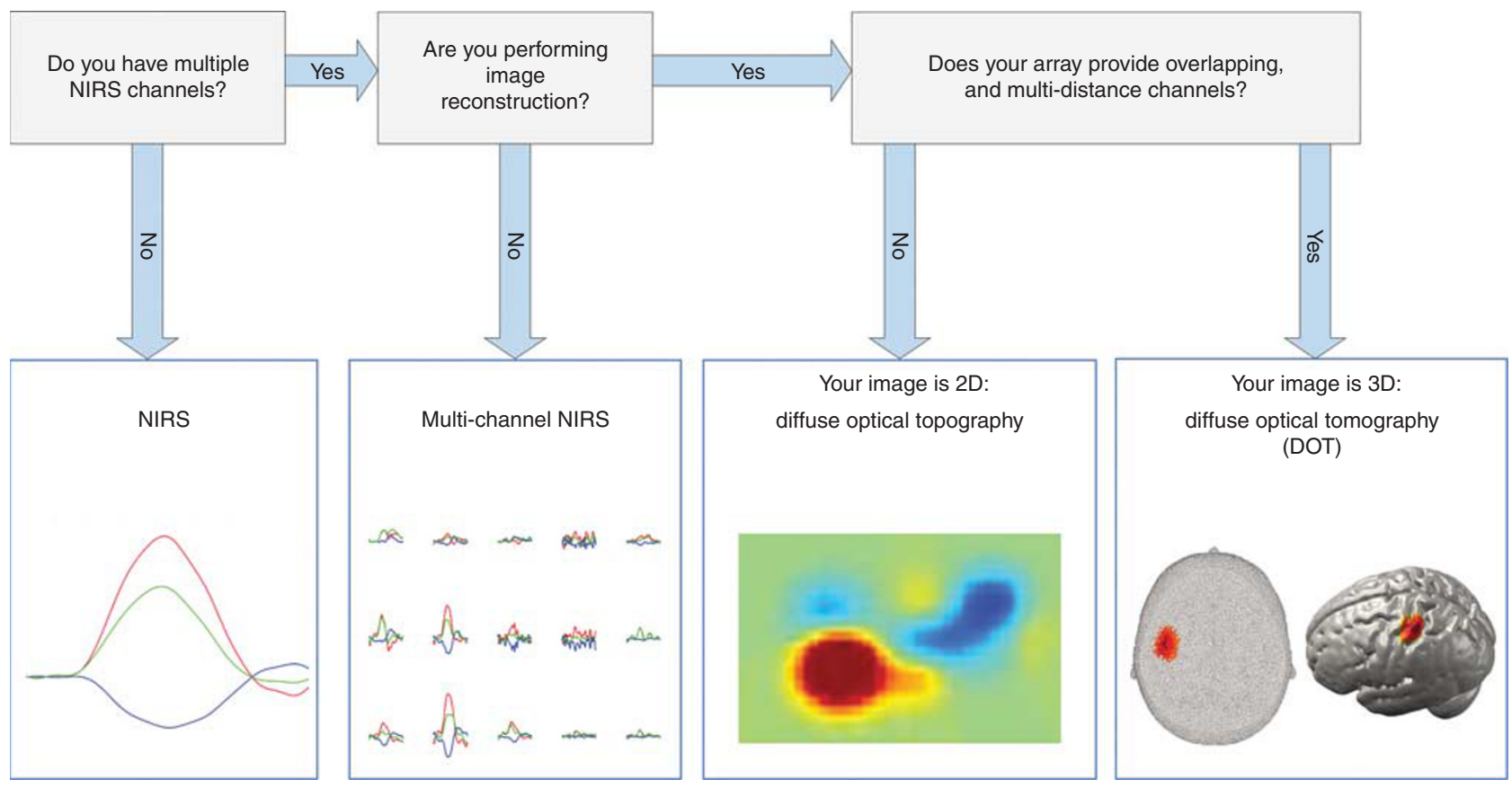

Figure 1. A decision tree that provides a definition of the different forms of diffuse optical monitoring. Although nomenclature varies across the field, these are the definitions preferred by the authors. 2D, two dimensional; NIRS, near-infrared spectroscopy.

deoxyhemoglobin, the technique can be used to measure the functional hemodynamic response to specific stimuli. This technique is known as functional NIRS or fNIRS. The same physiological principles exploited by fNIRS underpin the blood-oxygen-level-dependent signal, which is used in functional magnetic resonance imaging (fMRI). However, although the blood-oxygen-level-dependent signal is predominantly sensitive to the concentration of deoxyhemoglobin (9), fNIRS methods have the advantage of being able to monitor the concentrations of both oxyhemoglobin and deoxyhemoglobin independently, which significantly aids the physiological interpretation of fNIRS measurements.

In infants, the maturity of neurovascular coupling is still in question, and studies have yielded adult-like responses (10-12), inverted (decreased oxyhemoglobin and increased deoxyhemoglobin) responses (13), or a mixture of the two $(14,15)$. Although the nature of this response remains controversial (and is beyond the scope of this review), hemodynamic changes that are temporally correlated to the external stimulus can still provide an indication of neural activation, and the interpretation of response polarity should be carefully assessed depending on the nature of the subject or study.

\section{Early Application of fNIRS in Infants}

Early infant functional studies typically used a single source and detector providing one channel-i.e., one measurement of the cortical changes-in a primary area of interest $(14,16,17)$. However, the majority of commercial fNIRS systems now use numerous source and detector fibers into an array that provides multiple fNIRS channels facilitating the examination of extended cortical regions. The most widely used multi-channel systems are continuous-wave (CW) devices, which are relatively low-cost and simple to set up (for review of CW systems, see (ref. 18)).

Multi-channel CW systems measure light attenuation by continuous illumination of the head with multiple sources. To distinguish which source gave rise to the light measured at any given detector, either time or frequency multiplexing methods must be used. In time multiplexing, each source is illuminated in turn for a specific period of time, so that the origin of any detected light is always apparent. In frequency multiplexing, all the sources are illuminated continuously, but with each source modulated at a distinct frequency. CW systems typically allow a sampling rate from 5 to $100 \mathrm{~Hz}$, providing accurate temporal information on cortical hemodynamic activity (19).

\section{Two-Dimensional Mapping and Optical Topography}

The simplest approach to obtaining images of spatial variation of brain activity using NIR methods is to create a twodimensional map of the responses obtained using multichannel CW fNIRS devices. These two-dimensional representations can be generated by interpolating the signal measured by multiple fNIRS channels across the interrogated area $(20,21)$. This approach is often referred to as optical topography. Although this technique has helped accelerate our understanding of neurodevelopment and cognition in the infant brain, including language (22-25), vocal $(26,27)$, social (28), and face (29) perception (for more reviews, see 

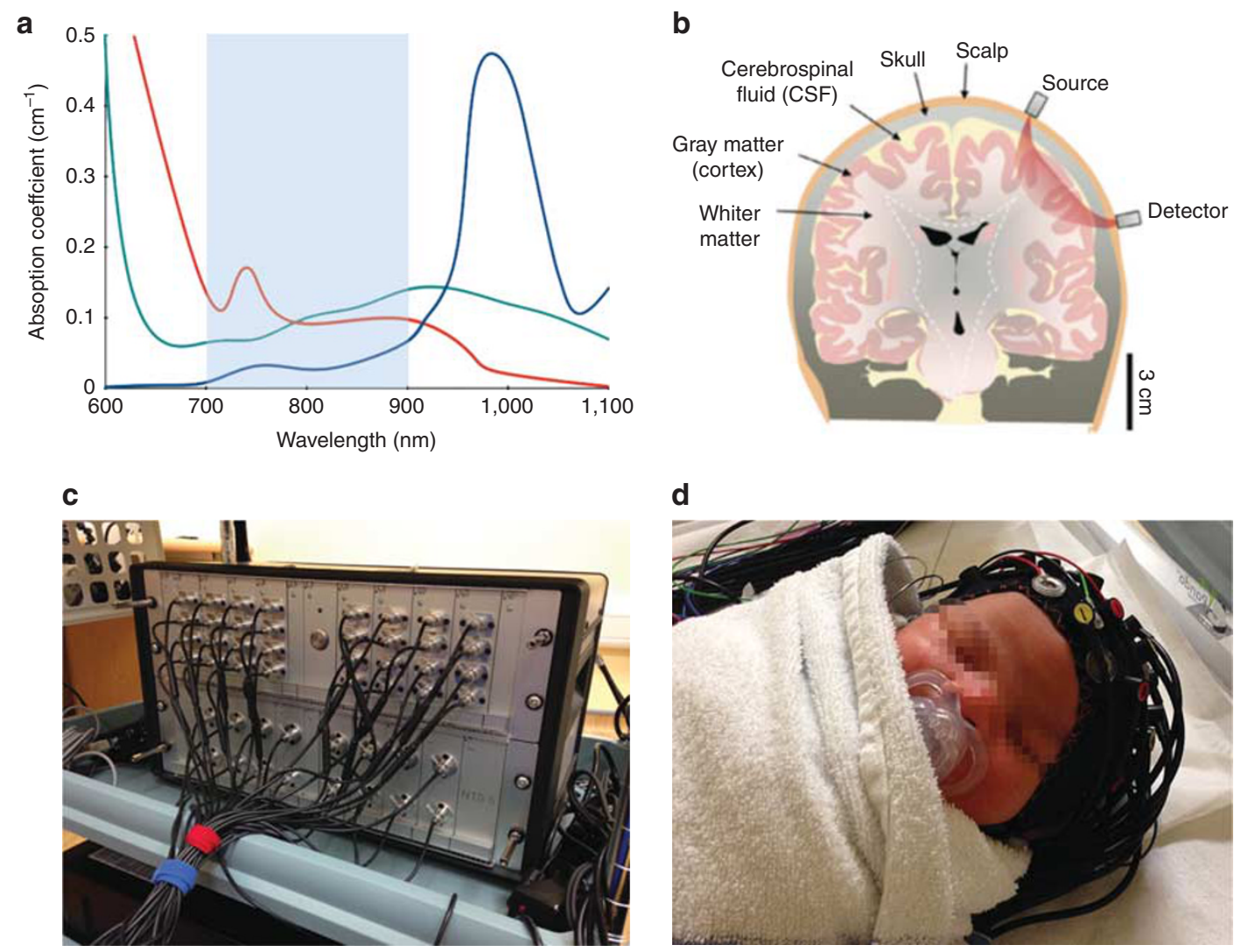

Figure 2. Principles and practical aspects of diffuse optical tomography. (a) The absorption properties of oxyhemoglobin (red line), deoxyhemoglobin (green line), and water (blue line) in biological tissue. In the optical window (shaded in blue) between 700 and $900 \mathrm{~nm}$, light is relatively transparent as the absorption by water molecules is relatively low compared with oxyhemoglobin and deoxyhemoglobin. (b) The path of near-infrared light from an optical source follows a banana shape, as it travels in cerebral tissue before the transmitted light is measured at the detector (84). (c) The UCL Optical Imaging System (Gowerlabs, London, UK). This is a continuous-wave device that samples at $10 \mathrm{~Hz}$ and provides 16 optical sources and 16 detectors. (d) An example of headgear design used in infant studies of whole-head diffuse optical tomography.

(refs 30-33)), the anatomical registration and spatial resolution of the resulting images is limited, and the quantitative accuracy of the images can be compromised (34). It is important to note, however, that significant functional information can be obtained without absolute quantification; fMRI is rightly considered the gold standard in functional neuroimaging, but in most common applications it remains purely qualitative (35).

\section{DIFFUSE OPTICAL TOMOGRAPHY}

DOT enables the production of three-dimensional images. The term "diffuse" describes the path of NIR light when it enters tissue (as explained in the "Principles of Optical Imaging" section). Unlike two-dimensional topographic approaches, DOT exploits measurements from multiple overlapping channels, with a range of source-detector distances to obtain depth information. A major advantage of this approach is the ability to separate hemodynamics occurring at different depths, and thus reduce the influence of blood oxygenation changes in extracerebral tissues such as the scalp or skull (see section "Physiological Signal Contamination").
The quality of DOT images is dependent on the number of channels that sample a given volume of tissue. The total number of channels one can achieve is dependent on the size and weight of the optical fibers and the number of source and detectors available in a DOT system. Although a sparse array can provide whole-head coverage (36), the resulting spatial resolution can be compromised. At present, commercial devices that can perform DOT (Figure 2c) provide 10-20 sources and 10-20 detectors, which allow imaging of subsections of the adult cortex or the majority of the infant cortex. Image resolution is a function of array density, such that dense arrays provide better spatial resolution (37). However, no DOT system (commercially available or otherwise) has yet been demonstrated that can provide dense sampling (i.e., with a nearest-neighbor source-detector separation of $<15 \mathrm{~mm}$ ) over the whole scalp of either the infant or the adult (38).

\section{DOT Image Reconstruction}

DOT image reconstruction is an example of an inverse problem, in which the goal is to determine internal features of an object using measurements of light transmitted between 
a

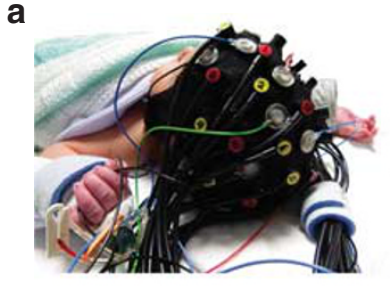

b

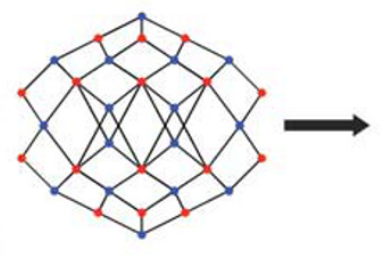

d
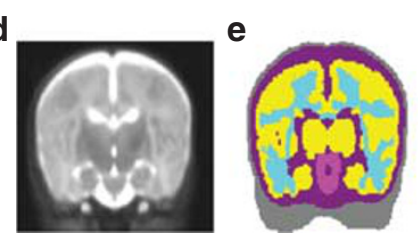

$\mathbf{f}$

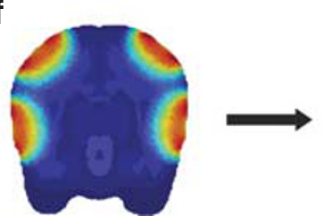

C

g

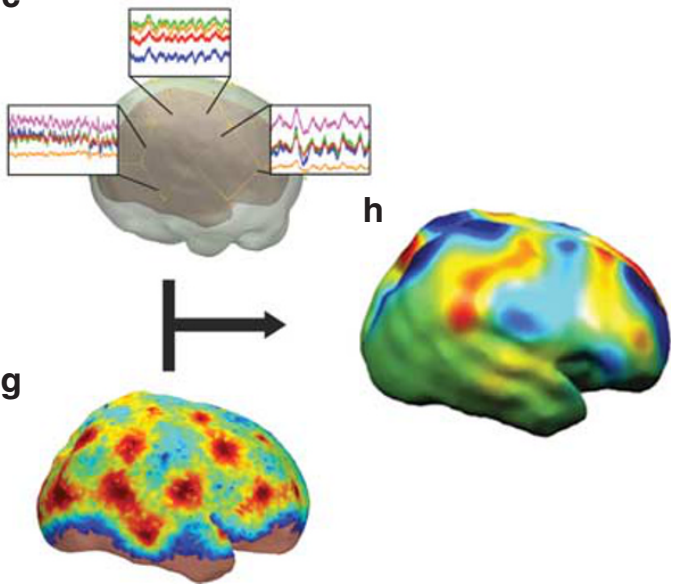

Figure 3. Diffuse optical tomography image reconstruction. (a) A photo of the headgear used in infant whole-head diffuse optical tomography scanning (36). (b) A schematic of the locations of sources (red dots) and detectors (blue dots) of the headgear in a (36). The black lines represent source-detector channels. (c) During scanning, raw light intensity data are converted to changes in optical density and these are assigned to the location of the source-detector channels of the headgear array. (d) An anatomical magnetic resonance imaging (MRI) is required to provide structural information for image reconstruction. This can either be the infant's own MRI or an age-matched atlas may be used. (e) The anatomical MRI image is segmented to identify the different tissue types (such as scalp, cerebrospinal fluid, gray matter, and white matter), as these will have different optical properties (74). (f) A mathematical model of how light will travel through brain tissue is derived (74). This will define how light intensity measured at a detector will change given localized changes in oxyhemoglobin and deoxyhemoglobin. (g) A sensitivity matrix describing how the changes in light intensity at each channel location (from (b)) relate to the changes in oxyhemoglobin and deoxyhemoglobin concentration (the final desired image) can be calculated using the model of light propagation in tissue from (f). (h) The desired image is reconstructed by combining the properties of the sensitivity matrix $(\mathbf{g})$ with the channel data (c).

points on the surface. DOT image reconstruction is ill-posed and highly underdetermined. Ill-posed means that many different internal configurations could equally explain the measurements made at the surface (i.e., there is no unique solution), and highly underdetermined means that the number of unknowns (i.e., voxels in our image) far exceeds the number of measurements made at the surface. One approach to simplify this problem is to produce images of a difference between two points in time, rather than to attempt to create images of the absolute optical properties of the brain. By assuming that the changes that have occurred between these two time points are small relative to the background optical characteristics of the tissue, the DOT reconstruction problem can be linearized, significantly simplifying the mathematical challenge (39). A schematic of DOT image reconstruction is shown in Figure 3 and for a more complete summary see (refs 39-41).

\section{APPLICATION OF DOT IN THE NEWBORN BRAIN}

The earliest infant DOT images were reconstructed in the late 1990s. For example, hemodynamic changes over the sensorimotor regions were demonstrated in the premature infant cortex by Chance et al. (42) using a system consisting of nine sources and four detectors. Hintz et al. (13) reconstructed functional images of passive arm movements over the motor cortex in preterm infants using an array with 9 sources and 16 detectors providing 144 independent measurements or channels.
More recently, the neonatal application of DOT has become more widespread. In a study of healthy newborn infants, Liao et al. (43) used a CW high-density DOT system to image functional activation of the primary visual cortex. To counteract the effect of superficial hemodynamic signals from the extracerebral tissue, superficial signal regression between the first- and second-nearest source-detector paired channels was carried out. Bilateral increases in oxyhemoglobin concentration were apparent in response to visual stimuli in the reconstructed DOT images. A direct comparison of the hemodynamic response in the DOT images was carried out with the conventionally recorded hemodynamic changes in the source-detector channels. This demonstrated an increase in the signal-to-noise ratio of the DOT images by twofold compared with the channel-wise data.

Intrinsic brain activity at rest in the absence of a stimulus can also provide information on functional cerebral development. This field, originally established using the bloodoxygen-level-dependent signal in fMRI research, is known as resting-state functional connectivity (44), and resting-state functional connectivity networks characterized by bilateral cortical connectivity have been described in the term and preterm infants (45-48).

Focusing on the visual cortex, White et al. (49) produced the first DOT images of bilateral occipital connectivity at rest in preterm- and full-term-born infants. An array consisting of 18 sources and 16 detectors was placed over the occipital cortex to record resting-state brain activity for up to $20 \mathrm{~min}$. To identify connectivity, two standard approaches, seed-based 

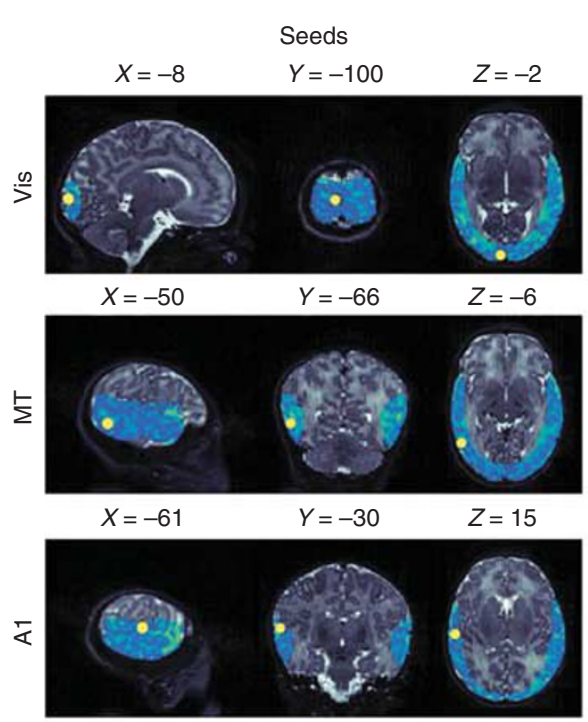

fcDOT $\left(\Delta \mathrm{HbO}_{2}\right)$
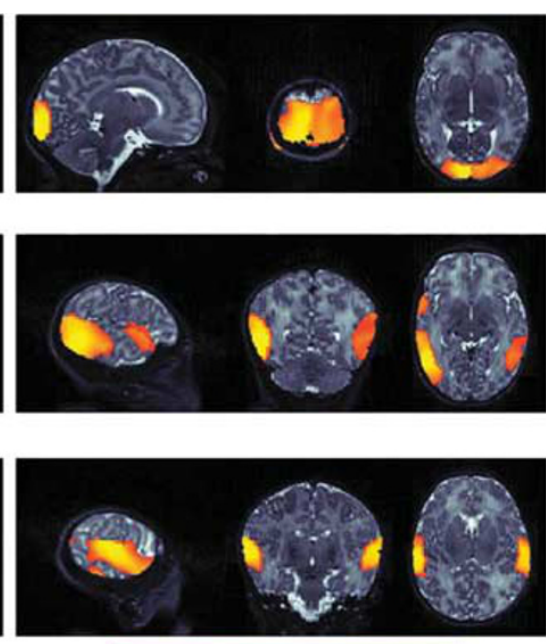

0.2
fcMRI (BOLD)
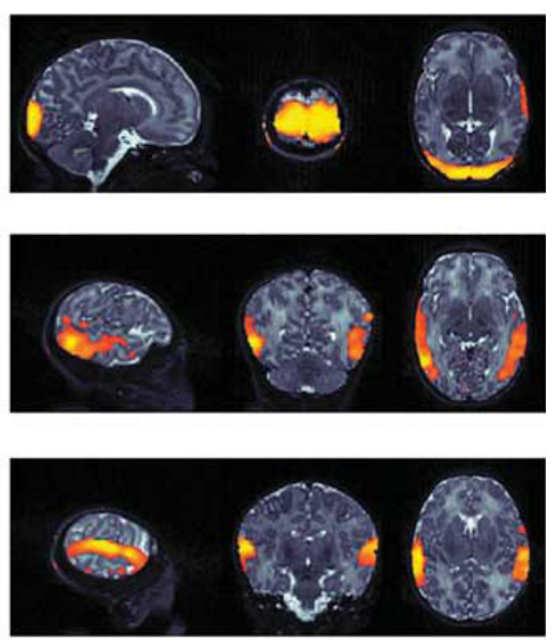

Figure 4. Resting-state functional connectivity maps from a single healthy term infant (38). The first column indicates the regions of interest selected for seed-based analysis of the visual (vis), middle temporal (MT), and primary auditory (A1) networks. The second column displays the correlation maps (i.e., networks) for each region of interest from functional connectivity diffuse optical tomography (fcDOT). The oxyhemoglobin signal $\left(\Delta \mathrm{HbO}_{2}\right.$ ) from DOT is overlaid on the subject-specific anatomical magnetic resonance imaging (MRI) image. The third column displays the correlation maps for each region of interest using the blood-oxygen-level-dependent (BOLD) signal in functional MRI (fcMRI).

analysis and independent component analysis, were carried out on the DOT images. Visual networks with bilateral connectivity between both cerebral hemispheres in the visual cortex were identified in the full-term and healthy preterm groups. Interestingly, one preterm subject with a diagnosis of a left occipital infarct displayed unilateral connectivity only demonstrating the sensitivity of DOT to cerebral pathology.

In a recent study, Ferradal et al. (38) also identified bilateral cortical connectivity in the resting state in healthy full-termborn infants using both fMRI and DOT imaging modalities in the same subjects for the first time. DOT resting-state data were recorded for up to $60 \mathrm{~min}$ using a dense array of 32 sources and 34 detectors over the temporal and occipital cortices. MRI scans were performed within a day following DOT scanning, and subject-specific structural MRI head models of light propagation were used. Using seed-based analysis and independent component analysis, the DOT images revealed bilateral connectivity in the visual, middle temporal, and primary auditory networks. Comparisons between the DOT and fMRI seed-based maps confirmed strong qualitative and quantitative agreement (Figure 4).

\section{APPLICATION OF DOT TO INVESTIGATE INFANT PATHOLOGY}

Perinatal hypoxic-ischemic encephalopathy (HIE) is a major cause of mortality and neurodevelopmental disability in term infants (50) resulting from severe perinatal deficit in cerebral blood flow and oxygen delivery (51). Subsequent brain injury is clinically characterized by seizure activity and abnormal neurological function, which may be associated with hemodynamic changes. DOT can offer valuable information on cerebral hemodynamics and oxygenation associated with brain injury, which are currently not available in the clinical setting. In a simultaneous electroencephalography (EEG) and DOT study of neurologically compromised infants, large transient hemodynamic events were identified in a small group of newborn infants diagnosed with clinical symptoms of seizures (52). Hemodynamic events were characterized by a slow increase in oxyhemoglobin, followed by a rapid decrease before a slow return to baseline (Figure 5).

In a case study using EEG and DOT, Singh et al. (36) reported the first DOT images of hemodynamic responses to electrographic seizures in an infant with severe HIE. A flexible head cap with 11 EEG electrodes and 16 sources and 16 detectors was used to record $60 \mathrm{~min}$ of data that contained seven electrographic seizure events lasting 30-90 s. Although there was spatial variation, the DOT images revealed an initial small increase in cortical blood volume, followed by a profound and extended decrease lasting several minutes (Figure 6).

In the absence of electrographic seizures, HIE infants may experience electrographic burst suppression, which is associated with poor neurodevelopmental prognosis (53). These patterns present as low-voltage electrical activity alternating with sharp high-voltage bursts that occur globally or in focal cortical regions (54). Neuroimaging strategies with improved spatial specificity compared with EEG can provide close monitoring of this pathological state. Using the same flexible DOT-EEG cap array (36), Chalia et al. (55) identified hemodynamic responses relating to electrographic burst 


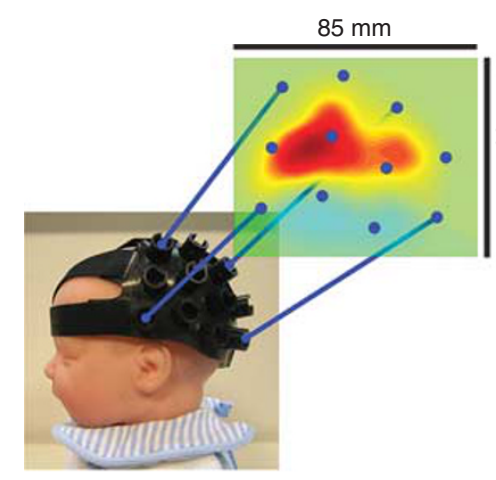

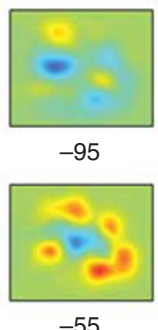

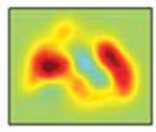

$-15$

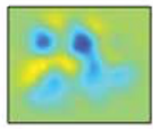

25
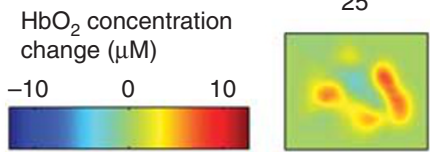

65

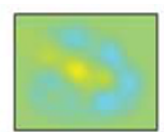

$-85$

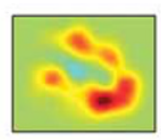

$-45$

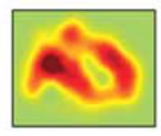

$-5$

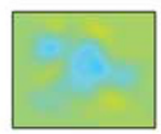

35

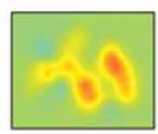

75

Time position (s)

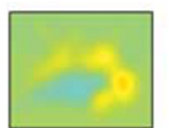

$-75$

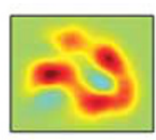

$-35$

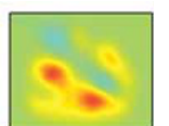

$-65$

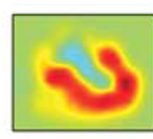

$-25$

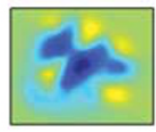

5

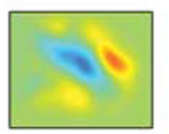

45

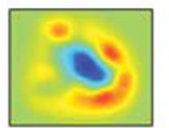

85

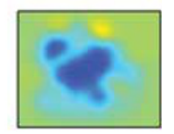

15

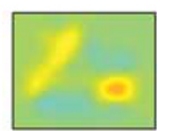

55

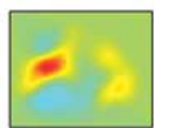

95

Figure 5. Diffuse optical tomography images reconstructed from a neurologically compromised infant for a single hemodynamic event (52). The series of images are reconstructed at a depth of 9-12 mm from the left lateral surface at $95 \mathrm{~s}$ preceding, to $95 \mathrm{~s}$ after the event. Red indicates an increase and blue a decrease in the concentration of oxyhemoglobin.

events of $4-6 \mathrm{~s}$, in six infants with HIE. The DOT images demonstrated a decrease in oxyhemoglobin before or during burst activity, followed by a large increase and a return to baseline with an undershoot. Patient-specific spatial patterns that were not apparent in the EEG were observed in the DOT images, signifying the complex morphology of burst suppression.

\section{TIME-DOMAIN DOT}

In addition to $\mathrm{CW}$ devices that measure only the changes in intensity of the detected light, and are thus unable to separate the effects of absorption and scatter or provide absolute quantification of tissue parameters, researchers have developed what are known as time-resolved or TD-DOT devices. TD devices operate by injecting very short pulses of light (picoseconds) into tissue and measuring the time of flight of individual photons using highly sensitive, photon-counting detectors (for review of TD methods, see (ref. 56)). By building up a histogram of photon flight times, TD-DOT provides a rich data set that contains information related to both the absorption and scattering properties of the underlying tissue. As a result, TD-DOT requires fewer assumptions to be made than CW methods, and it is possible to obtain absolute measurements of oxyhemoglobin and deoxyhemoglobin concentrations.

Because TD-DOT uses sensitive photon-counting detectors, it is also possible to make measurements over much larger source-detector distances including across the whole newborn infant head, allowing the creation of DOT images of the entire volume of the infant brain. Early images reconstructed using this technique were able to identify intracranial hemorrhages in preterm infants (57-59) (Figure 7). Compared with established imaging techniques such as ultrasound, blinded comparisons of DOT images in infants of varying gestation were interpreted as correct in six out of eight cases using TDDOT (60). Hemodynamic changes induced by adjusting mechanical ventilator settings in a full-term infant with HIE (61) have also been imaged across the whole brain, as have functional hemodynamic changes in the somato-motor cortex during passive arm movement in healthy preterm infants (62).

\section{CONTINUING CHALLENGES OF DOT}

Recent advances in DOT have allowed the technique to become an effective method for the investigation of both healthy and pathological neurodevelopment. However, as with all emerging technologies, a number of significant challenges still remain.

\section{Headgear Array Design}

Because of the physical size and weight of optical fibers, and the number of optodes available in a DOT system, the maximum number that can be used is currently limited, particularly for small infants. As a result, investigators using DOT must still choose between a sparse whole-head array (36) or a high-density localized array (38). The ability 


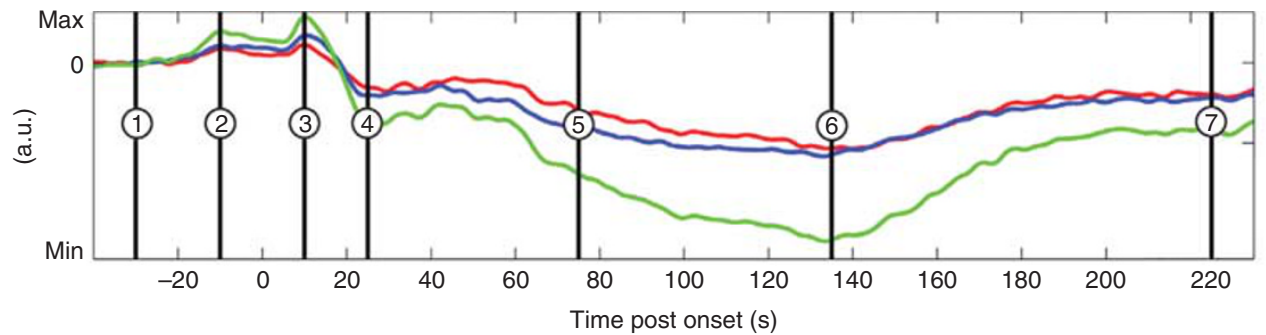

(1)
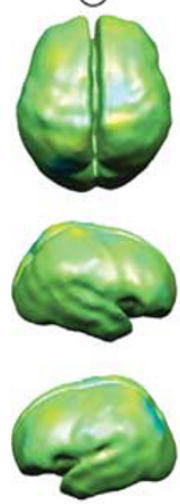

(2)
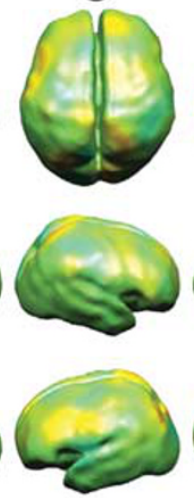

(3)
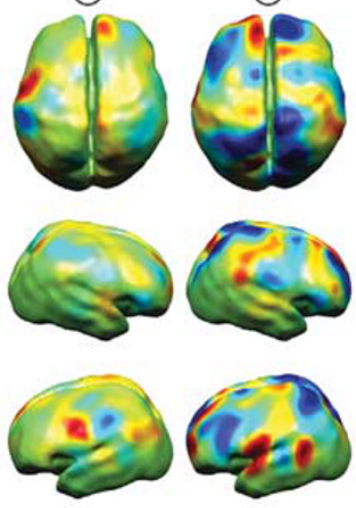

(4)
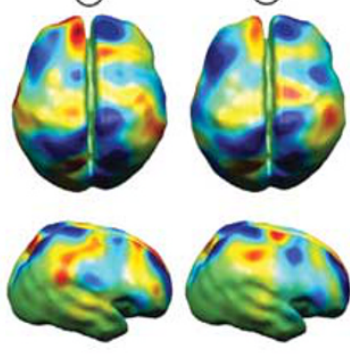

(5)
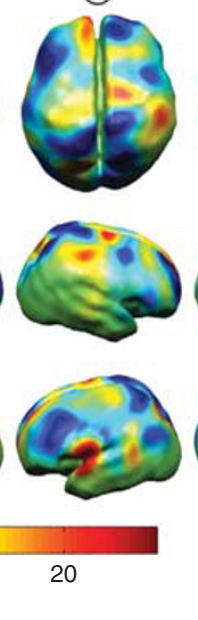

(6)
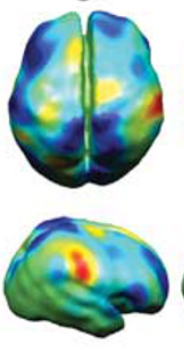

(7)
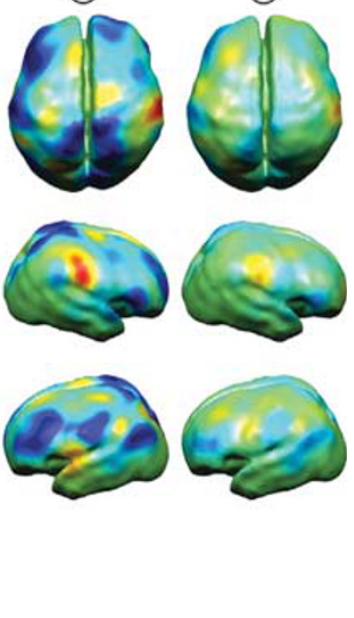

Figure 6. Diffuse optical tomography (DOT) images reconstructed in an infant with severe hypoxic-ischemic encephalopathy (36). The upper graph illustrates the oxyhemoglobin (red line), deoxyhemoglobin (blue line), and total hemoglobin (HbT, green line) changes for a seizure event identified by electroencephalography (EEG), which was recorded simultaneously. The numbered images below represent the cortical changes in three different views (dorsal, left, and right lateral) seen during the different time points of the seizure event as depicted in the graph. Red indicates an increase and blue a decrease in the HbT concentration in the DOT images.

a

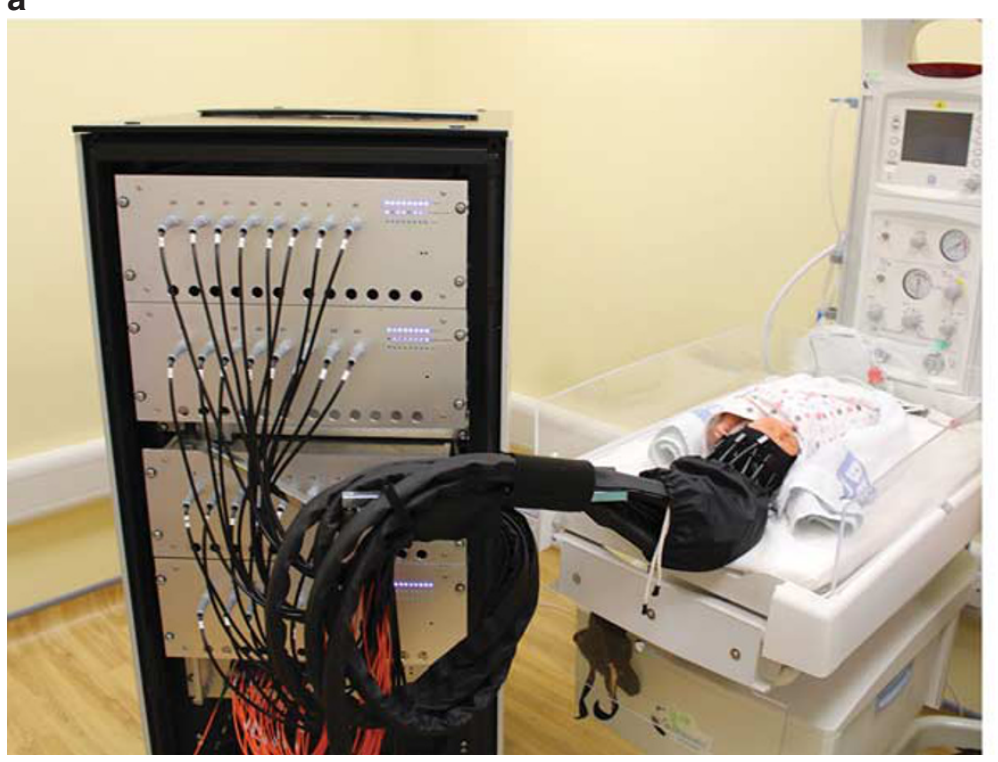

b

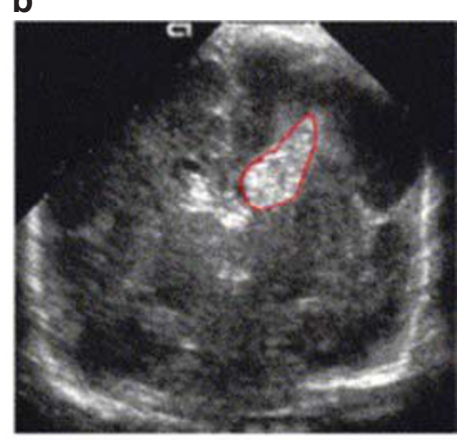

c

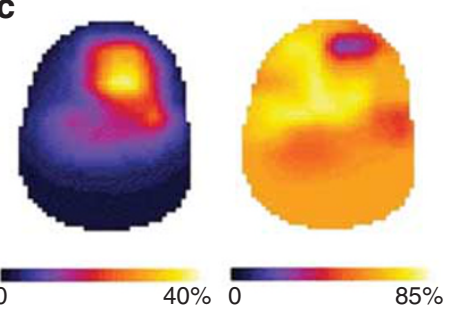

Figure 7. Time-domain diffuse optical tomography. (a) A photo of the UCL time-domain diffuse optical tomography (DOT) system (MONSTIR II) in the Rosie Hospital, Cambridge, UK (58). (b) A cranial ultrasound of a preterm infant revealing a left-sided intraventricular hemorrhage and hemorrhagic parenchymal infarct (58). (c) A three-dimensional DOT image taken at a coronal section of the same preterm infant showing regional blood volume (left) and oxygen saturation (right). There is an increase in hemoglobin concentration and decrease in oxygen saturation in the area corresponding to the hemorrhage and infarct. 
to flexibly design an array to suit a given experiment can be extremely beneficial, but the lack of a single 'catch-all' array solution does make it harder to perform quantitative crossstudy comparisons and also hinders the standardization and automation of data processing methods.

\section{Movement Artifacts}

Movement can be a significant problem in DOT studies, particularly in awake infants. Relative motion between the optical fiber and the scalp of the subject will affect optical coupling, which will often cause large variations in the measured optical intensity. Typically, the impact of movement on DOT data is to invoke transient, high-amplitude spikes in intensity, which immediately subside when the movement ends. Because these artifacts are temporary, periods of good-quality data DOT can usually be extracted, even in studies of awake infants. Unlike with fMRI, it is therefore rare that a whole DOT data set will have to be discarded because of movement artifacts. There are three broad strategies that can be used to address the problem of motion in DOT. The first is to prevent motion artifacts from occurring at all,by encouraging the infant to sleep,e.g., by feeding and swaddling the infant, and by ensuring that there are appropriate stimuli to engage an awake infant, as well as to construct the optode array headgear to optimize the coupling of the optodes to the head. The second is to exclude data that are deemed (by visual inspection or by the use of an accelerometer or video recording) to be contaminated by motion artifact, and the third is to use post-processing methods to clean the data to remove the effects of motion (63). In practice, most DOT studies use all three approaches, whereas NIRS and DOT motion artifact filtering methodologies continue to advance $(64,65)$.

\section{Physiological Signal Contamination}

One technical limitation common to all forms of NIRS and DOT is contamination of the measured signal by nonneuronal sources. These include systemic hemodynamic changes in both the brain and the superficial layers of the head including the skin caused by the cardiac cycle, blood pressure, and respiration. Because of the ability of DOT to obtain three-dimensional information, DOT image reconstruction can inherently separate the scalp and brain layers. Simple approaches used in NIRS can also be used for DOT, including low-pass filtering of the signal and block averaging to extract the signal that is temporally correlated to an external functional stimulus (18). More recent multivariate techniques, such as superficial signal regression (66-68), which uses short source-detector channels to measure scalp signal, and independent component analysis $(69,70)$ or principal component analysis (71-73), have been developed to isolate cerebral signals.

\section{Structural Priors}

A major advantage of DOT in comparison with fMRI is its portability. However, accurate DOT image reconstruction requires high-quality structural models of the subject's head to act as a prior for image reconstruction. Significant efforts have therefore been made to optimize an age-matched fourdimensional neonatal head model (74) using MRI head atlases as an alternative to subject-specific data $(36,49,55)$. An advantage of using a standard atlas is that it facilitates group comparisons across subjects, as it defines a common coordinate space (75), although this reduces the spatial specificity of the DOT images (76). Careful application of the optode array, and measurement of its position relative to certain cranial landmarks using a three-dimensional digitizer, can minimize the errors associated with registration and positioning and yield high-quality, anatomically registered DOT images.

\section{Statistical Analysis}

As an emerging field, there is no gold-standard approach for determining statistical significance or inference from DOT images as yet (for general review, see (ref. 77)). To extract the functional response to certain stimuli, event-related designs have used a general linear model approach to model the hemodynamic changes as a linear combination of independent variables (78). Similar to fMRI analysis, statistical parametric mapping tools that use a general linear model approach have also been developed and implemented for the statistical analysis of topographic (79) and tomographic images (80). However, there remain some concerns regarding the application of fMRI data processing methodologies to DOT because of the fundamentally different noise characteristics of the two data types (81). A major bottleneck in achieving a standardized (or even automated) DOT image reconstruction and processing pipeline is the lack of a 'catchall' high-density, whole-scalp imaging array.

\section{FUTURE DIRECTIONS}

One of the major confounding limitations of DOT methods remains the necessity of using large numbers of optical fibers. However, technology is rapidly developing that will allow DOT imaging without the use of optical fibers. Lightweight electronics can be worn by a subject with fewer cables in recent 'wearable' fNIRS systems enabling researchers to study mobile adult subjects $(19,82,83)$. Chitnis et al. (83) published the first functional images of the human brain obtained with a fiberless, high-density DOT system. Although designed for adults, this technology will likely be adapted for studies of the infant brain. By dispensing with the optical fibers, it may soon be possible to produce a wearable, high-density, whole-scalp DOT system that will provide high-quality functional images of the infant brain continuously at the bedside.

This has significant implications for both developmental neuroscience and clinical practice. For example, this would allow continuous monitoring of resting-state cortical connectivity in premature infants as a measurement of cognitive development or the detection of pathological cortical regions such as in stroke. 
Although these wearable technologies are impressive, they are still CW devices, and thus provide limited quantification. Historically, TD systems have been large and extremely expensive because of the complex electronics required for photon counting. The advent of low-cost, solid-state singlephoton avalanche detectors, which are now being developed for a range of novel time-of-flight technologies, will allow the issues of size and cost to be minimized in future generations of instruments (56). It is now reasonable to predict that fiberless TD-DOT technologies that provide quantitative, depth-sensitive, three-dimensional images of the whole infant brain with a resolution of $\sim 5 \mathrm{~mm}$ will be available within the next decade.

\section{CONCLUSIONS}

From the early measurements of cerebral oxygenation in newborns, to the more complex images of functional brain activity in recent years, DOT is rapidly emerging as an important tool for clinicians and neuroscientists to investigate the newborn brain. As the field has grown, so too has the expectation of the technology, and new challenges have arisen as we continue to push the boundaries of DOT. Wearable technologies are now emerging, which have the potential to allow continuous imaging of infant brain function at the cotside within the next few years. Innovations in TD-DOT technology imply that clinical TD-DOT systems are now on the horizon. As the technology advances, we must ensure that DOT is given the opportunity to become a clinically useful methodology; one that will provide clinicians with continuous measures of cerebral oxygenation, brain function, and neurodevelopment that will complement neonatal intensive care.

\section{ACKNOWLEDGMENTS}

This work was supported by the Medical Research Council, London, UK (MR/L017490/1), the Evelyn Trust, Cambridge, UK (09/26 2013 RTF), and the Engineering and Physical Sciences Research Council, London, UK (EP/ N025946/1).

Disclosure: The authors declare no conflict of interest.

\section{REFERENCES}

1. Jöbsis FF. Noninvasive, infrared monitoring of cerebral and myocardial oxygen sufficiency and circulatory parameters. Science 1977;198:1264-7.

2. van der Zee P, Cope M, Arridge SR, et al. Experimentally measured optical pathlengths for the adult head, calf and forearm and the head of the newborn infant as a function of inter optode spacing. Adv Exp Med Biol 1992;316:143-53.

3. Matcher SJ, Cope M, Delpy DT. Use of the water absorption spectrum to quantify tissue chromophore concentration changes in near-infrared spectroscopy. Phys Med Biol 1994;39:177-96.

4. Brazy JE, Lewis DV, Mitnick MH, et al. Noninvasive monitoring of cerebral oxygenation in preterm infants: preliminary observations. Pediatrics 1985;75:217-5.

5. Wyatt JS, Cope M, Delpy DT, et al. Quantification of cerebral oxygenation and haemodynamics in sick newborn infants by near infrared spectrophotometry. Lancet 1986;2:1063-6.

6. Wolf M, Greisen G. Advances in near-infrared spectroscopy to study the brain of the preterm and term neonate. Clin Perinatol 2009;36:807-34, vi.
7. Obrig $\mathrm{H}$, Villringer A. Beyond the visible-imaging the human brain with light. J Cereb Blood Flow Metab 2003;23:1-18.

8. Raichle ME, Mintun MA. Brain work and brain imaging. Annu Rev Neurosci 2006;29:449-76.

9. Ogawa S, Lee TM, Kay AR, et al. Brain magnetic resonance imaging with contrast dependent on blood oxygenation. Proc Natl Acad Sci USA 1990;87:9868-72.

10. Peña M, Maki A, Kovacić D, et al. Sounds and silence: an optical topography study of language recognition at birth. Proc Natl Acad Sci USA 2003;100:11702-5.

11. Taga G, Asakawa K, Maki A, et al. Brain imaging in awake infants by near-infrared optical topography. Proc Natl Acad Sci USA 2003;100: 10722-7.

12. Liao SM, Gregg NM, White BR, et al. Neonatal hemodynamic response to visual cortex activity: high-density near-infrared spectroscopy study. J Biomed Opt 2010;15:026010.

13. Hintz SR, Benaron DA, Siegel AM, et al. Bedside functional imaging of the premature infant brain during passive motor activation. J Perinat Med 2001;29:335-43.

14. Meek JH, Firbank M, Elwell CE, et al. Regional hemodynamic responses to visual stimulation in awake infants. Pediatr Res 1998;43:840-3.

15. Kotilahti $K$, Nissilä $I$, Näsi $T$, et al. Hemodynamic responses to speech and music in newborn infants. Hum Brain Mapp 2010;31: 595-603.

16. Bartocci M, Winberg J, Ruggiero C, et al. Activation of olfactory cortex in newborn infants after odor stimulation: a functional near-infrared spectroscopy study. Pediatr Res 2000;48:18-23.

17. Zaramella P, Freato F, Amigoni A, et al. 2001Brain auditory activation measured by near-infrared spectroscopy (NIRS) in neonates. Pediatr Res 49:213-9.

18. Scholkmann F, Kleiser S, Metz AJ, et al. A review on continuous wave functional near-infrared spectroscopy and imaging instrumentation and methodology. Neuroimage 2014;85 (Pt 1): 6-27.

19. Ferrari M, Quaresima V. A brief review on the history of human functional near-infrared spectroscopy (fNIRS) development and fields of application. Neuroimage 2012;63:921-35.

20. Maki A, Yamashita Y, Ito Y, et al. Spatial and temporal analysis of human motor activity using noninvasive NIR topography. Med Phys 1995;22: 1997-2005.

21. Franceschini MA, Toronov V, Filiaci M, et al. On-line optical imaging of the human brain with 160-ms temporal resolution. Opt Express 2000;6: 49-57.

22. Sato H, Hirabayashi $\mathrm{Y}$, Tsubokura H, et al. Cerebral hemodynamics in newborn infants exposed to speech sounds: a whole-head optical topography study. Hum Brain Mapp 2012;33:2092-103.

23. Minagawa-Kawai $\mathrm{Y}$, van der Lely $\mathrm{H}$, Ramus $\mathrm{F}$, et al. Optical brain imaging reveals general auditory and language-specific processing in early infant development. Cereb Cortex 2011;21:254-61.

24. Homae F, Watanabe H, Nakano T, et al. Large-scale brain networks underlying language acquisition in early infancy. Front Psychol 2011; 2:93.

25. Gervain J, Macagno F, Cogoi S, et al. The neonate brain detects speech structure. Proc Natl Acad Sci USA 2008;105:14222-7.

26. Lloyd-Fox S, Blasi A, Mercure E, et al. The emergence of cerebral specialization for the human voice over the first months of life. Soc Neurosci 2012;7:317-30.

27. Grossmann T, Oberecker R, Koch SP, et al. The developmental origins of voice processing in the human brain. Neuron 2010;65:852-8.

28. Lloyd-Fox S, Blasi A, Volein A, et al. Social perception in infancy: a near infrared spectroscopy study. Child Dev 2009;80:986-9.

29. Blasi A, Fox S, Everdell N, et al. Investigation of depth dependent changes in cerebral haemodynamics during face perception in infants. Phys Med Biol 2007;52:6849-4.

30. Lloyd-Fox S, Blasi A, Elwell CE. Illuminating the developing brain: the past, present and future of functional near infrared spectroscopy. Neurosci Biobehav Rev 2010;34:269-84. 


\section{Diffuse optical tomography in newborns $\quad$ Review}

31. Gervain J, Mehler J, Werker JF, et al. Near-infrared spectroscopy: a report from the McDonnell infant methodology consortium. Dev Cogn Neurosci 2011;1:22-46.

32. Aslin RN, Shukla M, Emberson LL. Hemodynamic correlates of cognition in human infants. Annu Rev Psychol 2015;66:349-79.

33. Vanderwert RE, Nelson CA. The use of near-infrared spectroscopy in the study of typical and atypical development. Neuroimage 2014;85 (Pt 1): 264-71.

34. Boas DA, Dale AM, Franceschini MA. Diffuse optical imaging of brain activation: approaches to optimizing image sensitivity, resolution, and accuracy. Neuroimage 2004;23(Suppl 1):S275-88.

35. Hoge RD. Calibrated FMRI. Neuroimage 2012;62:930-7.

36. Singh H, Cooper RJ, Wai Lee C, et al. Mapping cortical haemodynamics during neonatal seizures using diffuse optical tomography: A case study. Neuroimage Clin 2014;5:256-65.

37. Eggebrecht AT, Ferradal SL, Robichaux-Viehoever A, et al. Mapping distributed brain function and networks with diffuse optical tomography. Nat Photonics 2014;8:448-54.

38. Ferradal SL, Liao SM, Eggebrecht AT, et al. Functional imaging of the developing brain at the bedside using diffuse optical tomography. Cereb Cortex 2016;26:1558-68.

39. Arridge SR. Methods in diffuse optical imaging. Philos Trans A Math Phys Eng Sci 2011;369:4558-76.

40. Hoshi Y, Yamada Y. Overview of diffuse optical tomography and its clinical applications. J Biomed Opt 2016;21:091312.

41. Hielscher AH, Bluestone AY, Abdoulaev GS, et al. Near-infrared diffuse optical tomography. Dis Markers 2002;18:313-7.

42. Chance B, Anday E, Nioka S, et al. A novel method for fast imaging of brain function, non-invasively, with light. Opt Express 1998;2:411-23.

43. Liao SM, Ferradal SL, White BR, et al. High-density diffuse optical tomography of term infant visual cortex in the nursery. J Biomed Opt 2012;17:081414.

44. Biswal BB, Van Kylen J, Hyde JS. Simultaneous assessment of flow and BOLD signals in resting-state functional connectivity maps. NMR Biomed 1997;10:165-70.

45. Doria V, Beckmann CF, Arichi T, et al. Emergence of resting state networks in the preterm human brain. Proc Natl Acad Sci USA 2010;107:20015-20.

46. Smyser CD, Inder TE, Shimony JS, et al. Longitudinal analysis of neural network development in preterm infants. Cereb Cortex 2010;20:2852-62.

47. Fransson P, Skiöld B, Engström M, et al. Spontaneous brain activity in the newborn brain during natural sleep-an fMRI study in infants born at full term. Pediatr Res 2009;66:301-5.

48. Fransson P, Skiöld B, Horsch S, et al. Resting-state networks in the infant brain. Proc Natl Acad Sci USA 2007;104:15531-6.

49. White BR, Liao SM, Ferradal SL, et al. Bedside optical imaging of occipital resting-state functional connectivity in neonates. Neuroimage 2012;59: 2529-38.

50. Lawn J, Shibuya K, Stein C. No cry at birth: global estimates of intrapartum stillbirths and intrapartum-related neonatal deaths. Bull World Health Organ 2005;83:409-17.

51. Volpe JJ. Perinatal brain injury: from pathogenesis to neuroprotection. Ment Retard Dev Disabil Res Rev 2001;7:56-64.

52. Cooper RJ, Hebden JC, O'Reilly H, et al. Transient haemodynamic events in neurologically compromised infants: a simultaneous EEG and diffuse optical imaging study. Neuroimage 2011;55:1610-6.

53. Hellstrom-Westas L, Rosen I. Electroencephalography and amplitudeintegrated EEG. In: Lagercrantz H, ed. The Newborn Brain: Neuroscience and Clinical Applications. Cambridge University Press: Cambridge, UK, 2010, pp 211-8.

54. Lewis LD, Ching S, Weiner VS, et al. Local cortical dynamics of burst suppression in the anaesthetized brain. Brain 2013;136:2727-37.

55. Chalia M, Lee CW, Dempsey LA, et al. Hemodynamic response to burstsuppressed and discontinuous electroencephalography activity in infants with hypoxic ischemic encephalopathy. Neurophotonics 2016;3:10.

56. Torricelli A, Contini D, Pifferi A, et al. Time domain functional NIRS imaging for human brain mapping. Neuroimage 2014;85 (Pt 1): 28-50.
57. Hebden JC, Gibson A, Yusof RM, et al. Three-dimensional optical tomography of the premature infant brain. Phys Med Biol 2002;47: 4155-66.

58. Austin T, Gibson AP, Branco G, et al. Three dimensional optical imaging of blood volume and oxygenation in the neonatal brain. Neuroimage 2006;31:1426-33.

59. Hintz SR, Benaron DA, van Houten JP, et al. Stationary headband for clinical time-of-flight optical imaging at the bedside. Photochem Photobiol 1998;68:361-9.

60. Hintz SR, Cheong WF, van Houten JP, et al. Bedside imaging of intracranial hemorrhage in the neonate using light: comparison with ultrasound, computed tomography, and magnetic resonance imaging. Pediatr Res 1999;45:54-9.

61. Hebden JC, Gibson A, Austin T, et al. Imaging changes in blood volume and oxygenation in the newborn infant brain using three-dimensional optical tomography. Phys Med Biol 2004;49:1117-30.

62. Gibson AP, Austin T, Everdell NL, et al. Three-dimensional whole-head optical tomography of passive motor evoked responses in the neonate. Neuroimage 2006;30:521-8.

63. Brigadoi S, Ceccherini L, Cutini S, et al. Motion artifacts in functional near-infrared spectroscopy: a comparison of motion correction techniques applied to real cognitive data. Neuroimage 2014;85(Pt 1):181-91.

64. Barker JW, Rosso AL, Sparto PJ, et al. Correction of motion artifacts and serial correlations for real-time functional near-infrared spectroscopy. Neurophotonics 2016;3:031410.

65. Gu Y, Han J, Liang Z, et al. Empirical mode decomposition-based motion artifact correction method for functional near-infrared spectroscopy. J Biomed Opt 2016;21:15002.

66. Gagnon L, Perdue K, Greve DN, et al. Improved recovery of the hemodynamic response in diffuse optical imaging using short optode separations and state-space modeling. Neuroimage 2011;56:1362-71.

67. Saager R, Berger A. Measurement of layer-like hemodynamic trends in scalp and cortex: implications for physiological baseline suppression in functional near-infrared spectroscopy. J Biomed Opt 2008;13: 034017.

68. Gregg NM, White BR, Zeff BW, et al. Brain specificity of diffuse optical imaging: improvements from superficial signal regression and tomography. Front Neuroenergetics 2010;2:14.

69. Markham J, White BR, Zeff BW, et al. Blind identification of evoked human brain activity with independent component analysis of optical data. Hum Brain Mapp 2009;30:2382-92.

70. Zhang H, Zhang YJ, Lu CM, et al. Functional connectivity as revealed by independent component analysis of resting-state fNIRS measurements. Neuroimage 2010;51:1150-61.

71. Franceschini MA, Joseph DK, Huppert TJ, et al. Diffuse optical imaging of the whole head. J Biomed Opt 2006;11:054007.

72. Zhang Y, Brooks DH, Franceschini MA, et al. Eigenvector-based spatial filtering for reduction of physiological interference in diffuse optical imaging. J Biomed Opt 2005;10:11014.

73. Virtanen J, Noponen T, Merilainen P. Comparison of principal and independent component analysis in removing extracerebral interference from near-infrared spectroscopy signals. J Biomed Opt 2009;14:054032.

74. Brigadoi S, Aljabar P, Kuklisova-Murgasova M, et al. A 4D neonatal head model for diffuse optical imaging of pre-term to term infants. Neuroimage 2014;100:385-94.

75. Ferradal SL, Eggebrecht AT, Hassanpour M, et al. Atlas-based head modeling and spatial normalization for high-density diffuse optical tomography: in vivo validation against fMRI. Neuroimage 2014;85(Pt 1): $117-26$.

76. Cooper RJ, Caffini M, Dubb J, et al. Validating atlas-guided DOT: a comparison of diffuse optical tomography informed by atlas and subjectspecific anatomies. Neuroimage 2012;62:1999-2006.

77. Tak S, Ye JC. Statistical analysis of fNIRS data: a comprehensive review. Neuroimage 2014;85(Pt 1):72-91.

78. Friston KJ, Holmes AP, Poline JB, et al. Analysis of fMRI time-series revisited. Neuroimage 1995;2:45-53. 
79. Ye JC, Tak S, Jang KE, et al. NIRS-SPM: statistical parametric mapping for near-infrared spectroscopy. Neuroimage 2009;44:428-7.

80. Hassanpour MS, White BR, Eggebrecht AT, et al. Statistical analysis of high density diffuse optical tomography. Neuroimage 2014;85(Pt 1): 104-6.

81. Huppert TJ. Commentary on the statistical properties of noise and its implication on general linear models in functional near-infrared spectroscopy. Neurophotonics 2016;3:010401.
82. Kassab A, Lan Jr JL, Vannasing P, Mohamad S. Functional near-infrared spectroscopy caps for brain activity monitoring: a review. Appl. Opt 2015;54:576-86.

83. Chitnis D, Airantzis D, Highton D, et al. Towards a wearable near infrared spectroscopic probe for monitoring concentrations of multiple chromophores in biological tissue in vivo. Rev Sci Instrum 2016;87:065112.

84. Hillman EM. Optical brain imaging in vivo: techniques and applications from animal to man. J Biomed Opt 2007;12:051402. 